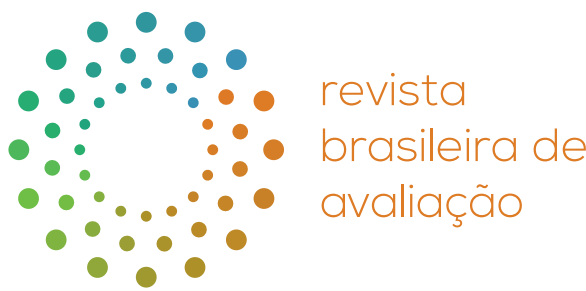

\title{
Avaliabilidade de coalizões para empoderamento de pessoas atingidas pela hanseníase
}

\section{Evaluability of coalitions to empowerment of people affected by hansen's disease}

Paula Soares Brandão ${ }^{1,2 *}\left[\right.$ branca] (1), Marly Marques da Cruz ${ }^{2}$ [preta] (1), Kátia Maria Braga Edmundo 3,4 [parda] (1)

${ }^{1}$ Universidade do Estado do Rio de Janeiro (UERJ), Departamento de Enfermagem em Saúde Pública, Rio de Janeiro, RJ, Brasil

${ }^{2}$ Fundação Oswaldo Cruz (Fiocruz), Escola Nacional de Saúde Pública Sergio Arouca (ENSP), Rio de Janeiro, RJ, Brasil

¿Universidade Estácio de Sá (UNESA), Rio de Janeiro, RJ, Brasil

${ }^{4}$ Centro de Promoção da Saúde (CEDAPS), Rio de Janeiro, RJ, Brasil

COMO CITAR: Brandão, Paula Soares, Cruz, Marly Marques da, \& Edmundo, Kátia Maria Braga (2021).

Avaliabilidade de coalizões para empoderamento de pessoas atingidas pela hanseníase. Revista Brasileira de Avaliação, 10(2), e101521. https://doi.org/10.4322/rbaval202110015

\section{Resumo}

A hanseníase é um problema de saúde pública para países e localidades com alta carga da doença e discriminação as pessoas atingidas. Monitorar e avaliar são desafios elucidados pela modelização de intervenções transformadoras da realidade. O objetivo é elaborar o Modelo Lógico Operacional (MLO) da formação de coalizões de pessoas atingidas pela hanseníase, pautado na avaliação de empoderamento. Trata-se de estudo de avaliabilidade usando Painel Delphi Político para consulta aos stakeholders. Os resultados compreendem o MLO da formação de coalizões como estratégia de participação para o empoderamento das pessoas atingidas, à escolha das perguntas avaliativas para compor o modelo teórico de avaliação e o envolvimento dos interessados de forma participativa. O estudo contribuiu para reflexão sobre a construção colaborativa da modelização, envolvimento de atores internacionais na formulação e implementação da intervenção e avaliação, limites e possibilidades da técnica de consenso à distância e fortalecimento de uma avaliação pautada na utilidade.

Palavras-chave: Avaliação em saúde. Empoderamento. Hanseníase. Participação dos interessados.

\begin{abstract}
Hansen's disease is a public health problem for countries and localities with a high burden of the disease and discrimination against those affected. Monitoring and evaluation are challenges elucidated by modeling interventions that transform reality. The objective is to develop the Logical Operational Model (MLO) for the formation of coalitions of people affected by Hansen's disease, based on the assessment of empowerment. This is an evaluability study using the Delphi Political Panel for consultation with stakeholders. The results include the MLO of coalition formation as a participation strategy for the empowerment of the affected people, the choice of evaluative questions to compose the theoretical model of evaluation and the involvement of stakeholders in a participatory way. The study contributed to reflection on the collaborative construction of modeling, the involvement of international actors in the formulation and implementation of intervention and evaluation, the limits and possibilities of the remote consensus technique and the strengthening of an evaluation based on utility.
\end{abstract}

Keywords: Health evaluation. Empowerment. Hansen's disease. Stakeholder participation.
A RBAVAL apoia os esforços relativos à visibilidade dos autores negros na produção científica. Assim, nossas publicações solicitam a autodeclaração de cor/etnia dos autores dos textos para tornar visível tal informação nos artigos.

Recebido: Julho 27, 2021

Aceito: Agosto 10, 2021

*Autor correspondente:

Paula Soares Brandão

E-mail: pbrandaofenf@gmail.com

\section{(c) BY}

Este é um artigo publicado em acesso aberto (Open Access) sob a licença Creative Commons Attribution, que permite uso, distribuição e reprodução em qualquer meio, sem restrições desde que o trabalho original seja corretamente citado. 


\section{Introdução}

A Hanseníase é uma enfermidade crônica, transmissível, curável, com características dermatoneurológicas e com possibilidade de produzir incapacidades físicas (Lastória \& Abreu, 2014). Por sua endemicidade em populações socialmente vulneráveis, pelo estigma associado e exclusão social, pelo pouco investimento em pesquisas, medicamentos e tecnologias para o cuidado é considerada uma doença negligenciada (Araujo et al., 2012; Dean et al., 2019). Para países e localidades, onde persiste alta carga da doença e discriminação associada ainda é um problema de saúde pública.

A Organização Mundial da Saúde (OMS) registrou 202.185 casos novos em 2019. As notificações concentraram-se em um grupo de 23 países, dos quais Índia, Brasil e Indonésia são responsáveis por mais de $80 \%$ da carga global da doença (WHO, 2020). A razão pela qual estes países não alcançaram a meta de eliminação da doença pode estar relacionada a não atuação efetiva sobre os determinantes sociais da hanseníase e a necessidade de implementar políticas intersetoriais.

Pescarini et al. (2018) e Nery et al. (2019) buscaram estabelecer relações entre os determinantes e marcadores de risco socioeconômico para o adoecimento por hanseníase. Para os autores, a persistência da endemia pode estar relacionada a fatores socioeconômicos como a pobreza, tipo e lotação das habitações (aumento das chances de convívio intradomiciliar com casos sem tratamento), saneamento básico (acesso a água potável e condições de higiene), baixo grau de escolaridade (acesso e compreensão de informações sobre a doença e manutenção do estigma), ocupação (tipo de trabalho e geração de renda), insegurança alimentar, acesso aos serviços de saúde e, ainda, ser do sexo masculino (visto a maior detecção precoce em mulheres).

Em 2009, o Brasil registrou 37.610 casos novos, dez anos depois foram reportados a OMS 27.863 casos novos da doença, ou seja, decréscimo de pouco mais de 10 mil casos. As regiões Centro-Oeste e Norte do país são aquelas que concentram a maior taxa de detecção, inclusive em menores de 15 anos, indicador considerado marcador epidemiológico para a transmissão e tendência da endemia (Brasil, 2020). Em 2019 foram registrados 1.545 casos nesta faixa etária, pouco menos de 1.000 casos, quando comparados aos 2.246 casos novos reportados em 2012 (WHO, 2021).

Quanto ao grau de incapacidade 2 (GIF2), indicador que contribui para análise operacional do programa, ocorreu uma pequena redução na taxa de casos novos diagnosticados com GIF2 entre os anos de $2009(12,72)$ e $2018(10,08)$, incidindo sobre as dificuldades existentes para realização de suspeição e diagnósticos precoces (Brasil, 2020). Deste modo, embora exista uma tendência de eliminação a nível nacional, persistem as disparidades regionais fruto das desigualdades sociais e da implementação da política para o controle da doença, principalmente, quanto a descentralização das ações para a atenção primária à saúde (Ribeiro et al., 2018).

Assim como no Brasil, desde o final da década de 1980, mundialmente vários esforços foram adotados para alcançar a meta de eliminação, ou seja, prevalência menor que um caso de Hanseníase para cada 10.000 habitantes (Ribeiro et al., 2018). O planejamento e as ações para o controle da doença foram organizados através de estratégias globais e planos nacionais com foco no fortalecimento do uso da poliquimioterapia, detecção de casos novos de modo oportuno e precoce, integração dos serviços de saúde e redução de incapacidades físicas decorrentes da doença. Apesar da diminuição do número de casos ao longo dos anos, estas ações não foram suficientes para reduzir a discriminação e promover a inclusão das pessoas atingidas e suas famílias, obrigando a OMS a repensar as estratégias adotadas.

A "Estratégia Global 2016-2020: Aceleração rumo a um mundo sem Hanseníase" foi organizada em três pilares, onde o primeiro versa sobre o fortalecimento do controle, coordenação e governo, o segundo trata sobre como combater a Hanseníase e suas complicações e o terceiro se refere ao combate a discriminação e a promoção da inclusão. $O$ terceiro pilar considera os aspectos humanos e sociais como parte da política, através da abordagem de todas as formas 
de discriminação e estigma, do empoderamento, da participação social e da eliminação de leis e/ou práticas discriminatórias (WHO, 2016).

Para compreender e analisar os mecanismos de participação das pessoas atingidas pela hanseníase que geram empoderamento faz se necessário ampliarmos o escopo para além do programático. Deste modo, as coalizões como estratégia de participação para empoderamento são aqui compreendidas como as articulações entre de organizações de pessoas atingidas pela hanseníase e suas famílias, atores da sociedade civil organizada e governos em prol da garantia de direitos humanos, do nível local ao global (Anderson et al., 2015).

Em que pesem os esforços da OMS em estimular os Estados-membros a implantar ações voltadas ao terceiro pilar, o monitoramento e avaliação (M\&A) constituem-se como desafio. A ausência de um Modelo Lógico Operacional (MLO) orientador das intervenções e o uso de indicadores caracterizados pela execução ou não de determinada ação, quando comparados à experiência adquirida pelos Programas de Controle da Hanseníase para M\&A de indicadores epidemiológicos e operacionais, refletem a fragilidade da macro intervenção e a necessidade de modelização, considerando sua sustentabilidade e avaliação adequada.

Criar as condições para avaliação permite a produção de informações cientificamente válidas e legítimas socialmente para melhoria de uma intervenção a partir de um julgamento de valor (Champagne et al., 2011a). Nesse sentido, a Avaliação de Empoderamento visa o aumento da capacidade das partes interessadas na intervenção em planejar, implementar e avaliar seus próprios programas, de modo a atingir melhores resultados. Além disso, caracteriza-se por sua natureza político-emancipatória, engajamento dos stakeholders da concepção à conclusão da avaliação, o trabalho colaborativo e participativo, a incorporação de estratégias baseadas em evidências, o respeito ao saber local e o avaliador como um facilitador (Fetterman, 2015; Fetterman \& Wandersman, 2017).

As avaliações de empoderamento podem ser precedidas por um Estudo de Avaliabilidade (EA), sobretudo por valorizar o envolvimento das partes interessadas na etapa pré-avaliativa e pela tomada de decisão sobre o que avaliar. Este tipo de estudo permite a decisão sobre viabilidade, adequação da estratégia avaliativa, identificação prévia do contexto, disponibilidade de informações e utilidade da avaliação (Baratieri et al., 2019). A abordagem do EA contribui para a teoria da mudança, através do desenvolvimento e avaliação de um modelo lógico e da co-produção da avaliação entre pesquisadores e stakeholders (Brunner et al., 2019).

A realização de um EA exige delineamento metodológico. Baratieri et al. (2019) propõem à descrição do programa, a identificação das possíveis ou melhores perguntas avaliativas, a construção de um plano de avaliação e a produção de um acordo entre os interessados e envolvidos tanto na intervenção quanto na avaliação. O cumprimento destas etapas requer um rol de atividades, a exemplo da descrição do programa. Champagne et al. (2011a) sugerem a elaboração de Modelos Lógicos (causal, teórico ou operacional), de modo a apoiar o reconhecimento da lógica explícita pelo programa.

Modelizar uma intervenção é uma etapa essencial para as avaliações. De acordo com o tipo de avaliação, a modelização será capaz de exibir os vínculos entre a intervenção e seus efeitos, permitir que as melhores perguntas sejam feitas, esclarecer os objetivos a serem alcançados, testar a plausibilidade das hipóteses, apoiar a elaboração de quadro de desempenho, contribuir para a construção de instrumentos de medida adaptados e/ou auxiliar a tomada de decisões. Além disso, nas avaliações de implementação de programas a modelização poderá facilitar a compreensão da influência do contexto sob determinado aspecto da intervenção (Brousselle et al., 2011).

Este artigo tem por objetivo descrever o processo decisório, a partir de um estudo de avaliabilidade, para realização de uma pesquisa avaliativa sobre a formação de coalizões como estratégia de empoderamento de pessoas atingidas pela hanseníase. 


\section{Metodologia}

Trata-se de um Estudo de Avaliabilidade, no qual o método Delphi foi utilizado para consulta aos stakeholders sobre a elaboração do MC e MLT do terceiro pilar da estratégia global e do MLO da intervenção a ser avaliada. O Painel Delphi consiste em capturar o conhecimento e a experiência de especialistas sobre um determinado campo, separados geograficamente, para melhorar a tomada de decisão sobre um problema complexo (Marques \& Freitas, 2018). Optamos pelo Delphi Policy (DP) por permitir a identificação das opiniões divergentes na formulação ou implementação de políticas, estimular a criação de novas idéias e consultar um número maior de interessados (Oliveira et al., 2018). Deste modo, o EA dividiu-se em etapas pré-Delphi (organização das etapas propostas para o EA) e Delphi (aplicação dos instrumentos, análise das informações e devolução aos stakeholders).

A compreensão do pilar III "Combater a discriminação e promover a inclusão" foi necessária para execução do painel. Para tal, a modelização lógica seguiu as etapas propostas por Bezerra et al. (2010), a saber: a) identificação do problema que gerou a intervenção; b) reconhecimento da intervenção criada para solução do problema; c) objetivo geral; d) objetivos específicos; e) distinção das metas a serem alcançadas; f) indicadores propostos para medir as metas; g) identificação do público-alvo da intervenção; h) identificação dos componentes da intervenção a partir dos objetivos específicos; i) apontar as atividades propostas; j) indicar a estrutura necessária para que a intervenção ocorra; I) descrever os produtos e resultados que são esperados; $\mathrm{m}$ ) discernir os fatores que podem influenciar os resultados, para além dos relacionados à intervenção; n) coleta e análise das informações; e o) validade do modelo da intervenção.

As primeiras etapas (a-h) permitiram a visualização esquemática do problema que gerou a intervenção, das prováveis causas, possíveis consequências e dos atributos necessários para solução, constituindo-se como MLT (Figura 1). Em seguida, para cada componente, descrito no

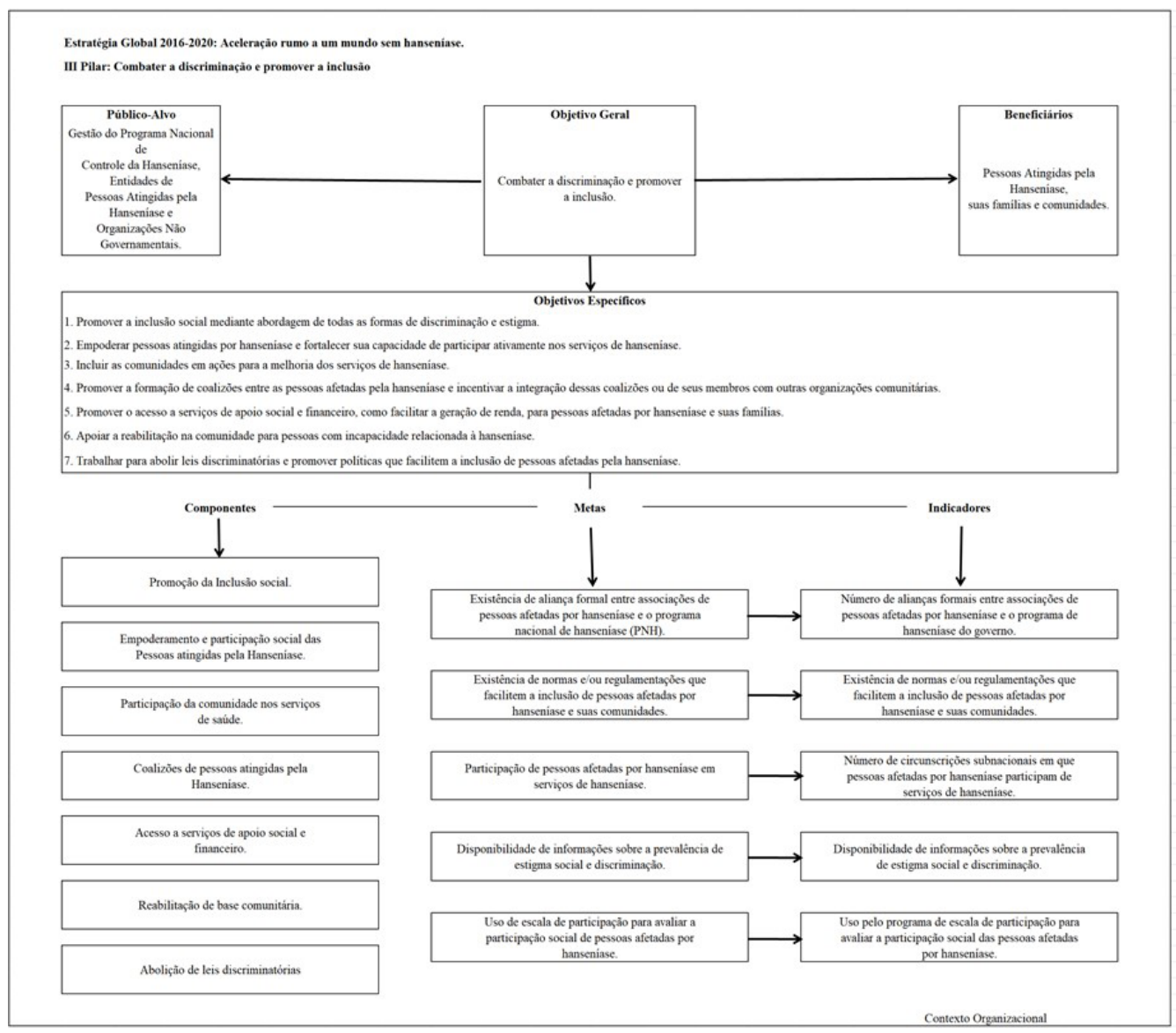

Figura 1. Modelo Teórico do III Pilar da Estratégia Global 2016-2020: Aceleração rumo a um mundo sem hanseníase. 
modelo e correspondente a um objetivo específico do terceiro pilar, foi elaborado um MLO. O MLT e os MLO foram pautados no documento base, no manual operacional, no guia de monitoramento e avaliação da Estratégia Global, no Guia para fortalecimento da participação das pessoas atingidas pela hanseníase nos serviços de saúde (WHO, 2011, 2016, 2017a, 2017b).

Os modelos foram criados seguindo a lógica pipeline (insumos, atividades, produtos, resultados e impacto) utilizando como ferramenta gráfica o software Lucidchart ${ }^{\circledR}$. A modelização não considerou o nível administrativo global e regional, apenas o nacional e local (estados/ províncias e/ou cidades), em virtude da viabilidade para operacionalização de uma avaliação. Além disso, o contexto foi dividido em ações para: todos os ambientes, ambientes com alta carga da doença e ambientes com baixa carga da doença. A diagramação inicial dos modelos pode ser exemplificada pelo MLO dos componentes "Empoderamento e Participação Social das pessoas atingidas pela hanseníase" (Figura 2) e "Coalizões de pessoas atingidas pela hanseníase" (Figura 3).

Os MLO dos sete componentes foram apresentados para atores chave, em uma reunião no I Encontro Latinoamericano e Caribenho de Entidades de Pessoas Atingidas pela Hanseníase, que aconteceu no Rio de Janeiro, em março de 2019. Nesta reunião, alguns atores declararam seu interesse na avaliação, porém sugeriram avaliar apenas um ou dois componentes, visto a complexidade da macrointervenção.

O DP foi realizado em três rodadas e respondido por um grupo de stakeholders previamente selecionados. Para cada rodada do Painel foi traçado um conjunto de ações, a saber: $1^{\text {a }}$ apresentação da proposta do EA, a modelização e verificação da lógica dos componentes, identificação do interesse dos stakeholders sobre os componentes e na avaliação, bem como priorização de um ou dois componentes a serem avaliados; $2^{\mathrm{a}}$ - apresentação das modificações propostas pelos stakeholders para os componentes eleitos e validação entre os participantes; e $3^{\mathrm{a}}$ - apresentação dos resultados e o MLO a ser avaliado na pesquisa avaliativa. Por conseguinte, para todas as rodadas foram construídos instrumentos de consulta de forma espelhada em português e inglês.

Para a primeira rodada, o instrumento de consulta para o Painel foi construído em Microsoft Excel, onde constavam abas destinadas: 1) à introdução; 2) às instruções aos participantes; 3) uma aba por componente contendo a figura da modelização lógica do componente, um quadro com doze perguntas para analisar a modelização, descrever dúvidas e recomendações; 4) ao ranqueamento dos componentes por interesse do participante na avaliação e 5) um quadro de análise dos interesses e governabilidade dos stakeholders na macrointervenção e na avaliação da mesma.

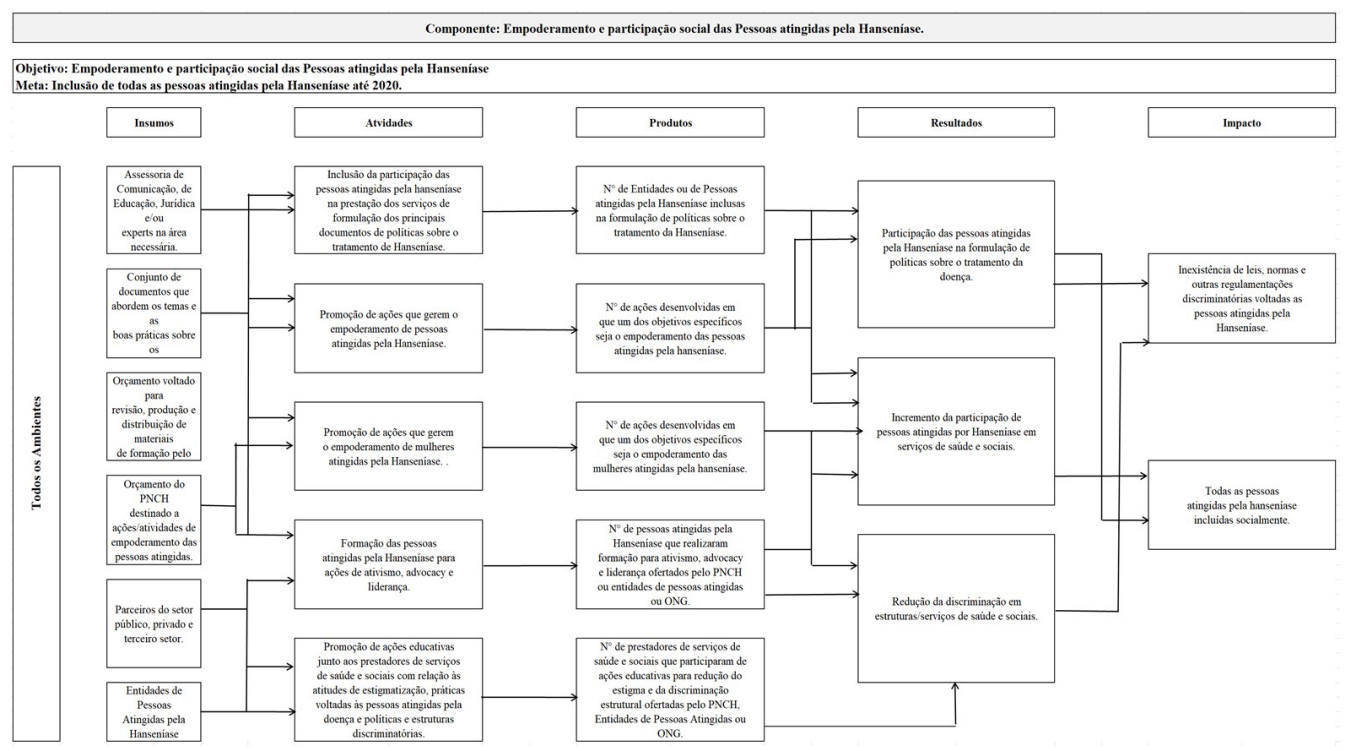

Figura 2. Modelo Lógico Operacional Empoderamento e Participação Social das pessoas atingidas pela Hanseníase. 


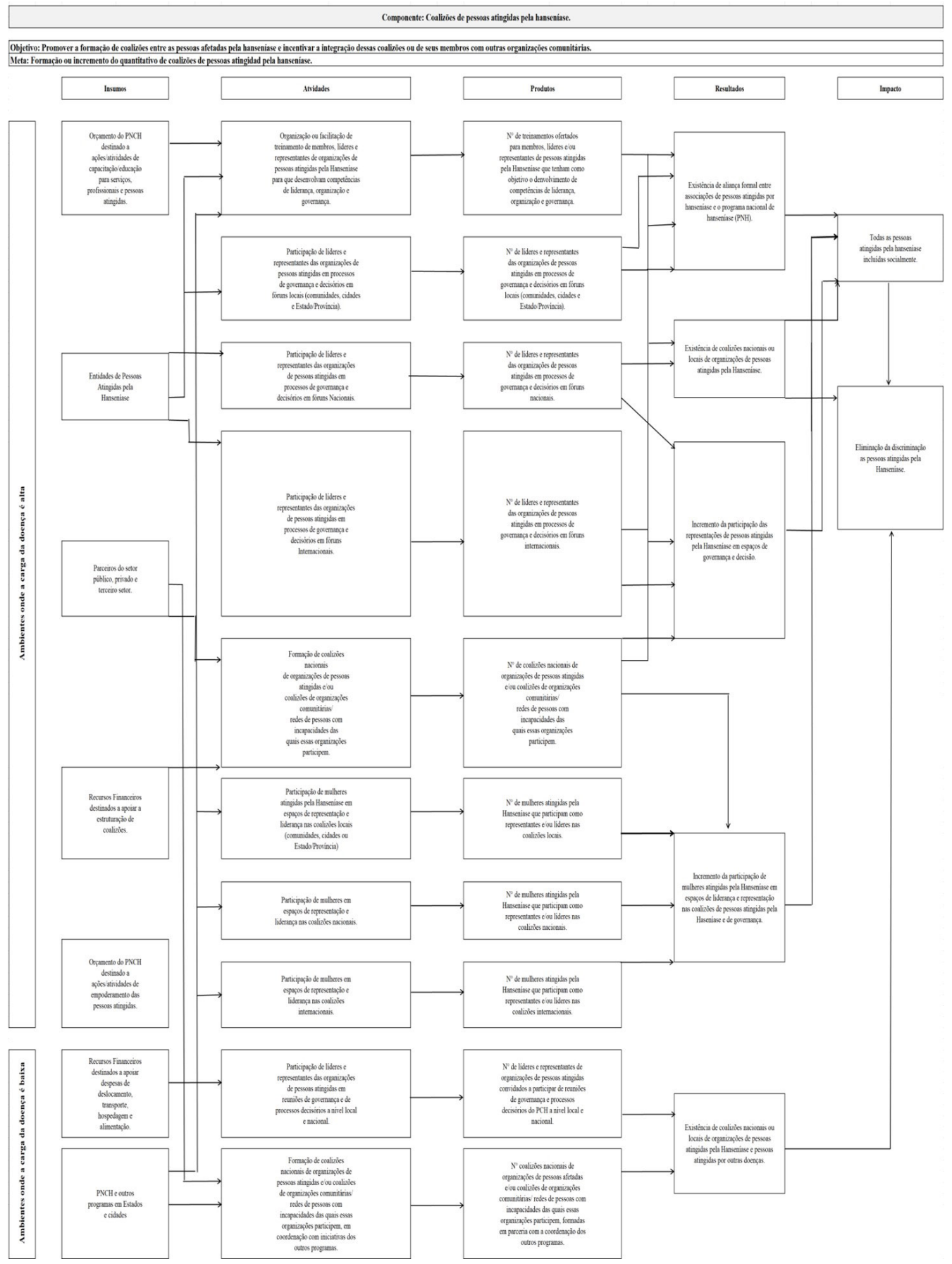

Figura 3. Modelo Lógico Operacional Coalizões de Pessoas Atingidas pela Hanseníase.

A análise das respostas utilizou técnicas de estatística simples e julgamento em quatro extratos sobre a adequação dos MLO, a saber: adequado (85-100\%), parcialmente adequado (50-84\%), pouco adequado (25-49\%) e inadequado (0-24\%). As críticas, recomendações e questionamentos foram organizados em um quadro e apoiaram a construção do novo modelo.

Para a segunda rodada foi elaborado um MLO e um questionário contendo quinze perguntas fechadas com opção de sugestão em texto livre para cada uma. O primeiro bloco de perguntas tratava sobre a adequação da modelização e o segundo bloco sobre a relação de possíveis perguntas avaliativas com objetivos de uma futura pesquisa avaliativa sobre o componente escolhido. Esta etapa foi elaborada em Microsoft Excel e na plataforma Google formulários. Todos os respondentes da primeira rodada receberam um relatório sobre as respostas e o novo MLO para subsidiar as respostas da segunda rodada. 
A aplicação do Delphi, como regra da técnica e seguindo os princípios éticos da Resolução n510/2016 para divulgação dos achados, garantiu o anonimato dos especialistas. A consulta aos stakeholdersse se deu no período entre abril e setembro de 2019. Encerrando-se com a apresentação do MLO para alguns participantes do Fórum Global de Entidades de Pessoas Atingidas pela Hanseníase realizado em Manila-Filipinas (setembro de 2019) e posterior devolução aos stakeholders dos resultados, constituindo-se como terceira e última rodada.

\section{Resultados}

O Modelo Causal e o Modelo Lógico Teórico foram previamente elaborados, de modo a representar a estrutura lógica entre as prováveis causas para discriminação e exclusão das pessoas atingidas pela Hanseníase e a teoria da mudança proposta pela "Estratégia Global 2016-2020: por um mundo livre de hanseníase". A partir destes modelos e da execução do painel Delphi Policy, tem-se como resultado a seleção e envolvimento das partes interessadas no estudo de avaliabilidade, o ranqueamento para escolha do componente a ser avaliado, as propostas de adequação da estrutura lógica dos MLO elaborados, a apresentação das principais críticas e sugestões dos modelos eleitos para avaliação e o MLO que servirá de base para a avaliação.

Os stakeholders foram inseridos a partir de sua atuação como lideranças ou tomadores de decisão, em entidades governamentais, não-governamentais, coalizão para pesquisa e de pessoas atingidas pela Hanseníase, engajadas na eliminação da doença como problema de saúde pública, tanto no cenário nacional quanto internacional. No total, foram selecionadas 12 organizações, entre elas 6 (seis) nacionais (Ministério da Saúde, três organizações não governamentais no Brasil que trabalham com pessoas atingidas, Sociedade Brasileira de Hansenologia e Morhan) e 6 (seis) internacionais (OMS, OPAS, ONU, International Federation Anti-leprosy Associations - ILEP, Fundação Nipon/Sasakawa, Global Partnership Zero Leprosy) e 24 representantes (8 do Brasil e 16 de outras nacionalidades).

Na primeira rodada, o painel foi encaminhado a todos os stakeholders e obteve resposta de $41,6 \%$. Na segunda foi enviado apenas para os respondentes da primeira, ou seja, dez especialistas e apenas um não respondeu. Na terceira rodada, considerada devolutiva, o MLO foi enviado aos participantes da segunda. Todas as respostas foram consideradas, em qualquer uma das etapas mesmo que incompletas, por se tratar de um Delphi Policy.

O grau de prioridade entre os componentes, para execução de futura pesquisa avaliativa, foi realizado através de ranqueamento. O ranqueamento foi construído em ordem crescente pelo número de vezes em que o grau de prioridade foi mencionado, tendo havido repetição de posicionamento por parte dos participantes e como resultado tivemos: $1^{\circ} \mathrm{Empoderamento} \mathrm{e}$ participação social das pessoas atingidas pela Hanseníase; $2^{\circ}$ Coalizões de pessoas atingidas pela Hanseníase; $3^{\circ}$ Acesso a serviços de apoio social e financeiro; $4^{\circ}$ Reabilitação de base comunitária; $5^{\circ} \mathrm{Participação} \mathrm{da} \mathrm{comunidade} \mathrm{nos} \mathrm{serviços} \mathrm{de} \mathrm{saúde;} 6^{\circ} \mathrm{Promoção} \mathrm{da} \mathrm{inclusão}$ social e $7^{\circ}$ Abolição de leis discriminatórias.

Os MLO por componente foram considerados parcialmente adequados, visto que pelo extrato de julgamento pontuaram para a pergunta sobre a completude da lógica do modelo entre 50\%-85\%. A depender do componente, algumas questões apresentaram maior fragilidade nos componentes estruturais - insumos e atividades, como a disponibilidade orçamentária (insumos) e interpretação de conceitos (atividades).

Entre o total de participantes, 30\% optaram por não responder as perguntas com sim ou não, e contribuíram frequentemente com críticas e/ou sugestões. Na maioria dos componentes, sugeriu-se a melhoria da relação gráfica das conexões (uso das setas) e a utilização de cores nas células para facilitar as relações entre os itens descritos.

Este processo resultou na elaboração de um único MLO desenvolvido a partir da segunda rodada do Painel Delphi Político e contemplando a sugestão dos interessados em avaliar um ou dois componentes apenas. O MLO “Formação de coalizões como estratégia de participação para o empoderamento das pessoas atingidas pela Hanseníase" considerou as críticas, 
recomendações e questionamentos aos modelos dos componentes "Empoderamento e Participação Social das pessoas atingidas pela Hanseníase" e "Coalizões de pessoas atingidas pela Hanseníase" (Quadro 1).

Na primeira rodada apenas três stakeholders preencheram o quadro sobre papel e interesse na avaliação, o que gerou a necessidade de reavaliar este objetivo para validação na segunda rodada. Os respondentes relataram dificuldades em compreender a metodologia para elaboração dos MLO apresentados no painel, visto que algumas temáticas não são possíveis de serem expressas com clareza em um modelo pipeline. Além disso, a complexidade dos modelos, o número de abas e perguntas a serem respondidas tornou o processo cansativo levando alguns a optarem pelos modelos de maior interesse ou facilidade de compreensão. Este evento contribuiu para repensar a organização da segunda rodada.

A elaboração do novo MLO foi feita a partir do ranqueamento de interesse dos stakeholders e das contribuições através das críticas e sugestões. O modelo considerou a Promoção da Saúde quanto ao seu papel de capacitação, advocacia para a saúde e mediação, conforme preconizado na Carta de Ottawa, e os conceitos da Classificação Internacional de Funcionalidades, Incapacidades e Saúde (CIF) propostos pela OMS (1986, 2013). A participação e o empoderamento foram divididos em níveis (individual, da comunidade e social). Além disso, partimos a princípio de que participação e empoderamento são retroalimentados e que a formação de coalizões, o controle social, leis e práticas são estratégias de participação a serem consideradas foco da avaliação. A partir das críticas ao modelo pipeline buscou-se a formulação de uma nova lógica considerando a estrutura, o processo, os resultados e os contextos.

O MLO “Formação de coalizões por pessoas atingidas pela Hanseníase" (Figura 4) é o resultado da segunda rodada e difere do apresentado pelos seguintes achados do Painel: a) inclusão de situações de participação individual que não estavam presentes no primeiro modelo, b) compreensão da interseção entre participação da comunidade e social por meio do tipo de associações, c) foco na formação de coalizões, d) acréscimo de estruturas sugeridas pelos stakeholders, e) diferenciação entre participação da comunidade e social, em virtude do Brasil possuir a participação social institucionalizada no Sistema Único de Saúde (SUS), e f) o acréscimo do empoderamento organizacional nos resultados, de modo a garantir o M\&A das coalizões.

Os stakeholders definiram como perguntas avaliativas para o modelo: a) "A formação de coalizão foi implementada de forma a garantir o empoderamento individual, da comunidade, organizacional e social das pessoas atingidas pela Hanseníase?", b) "As ações adotadas

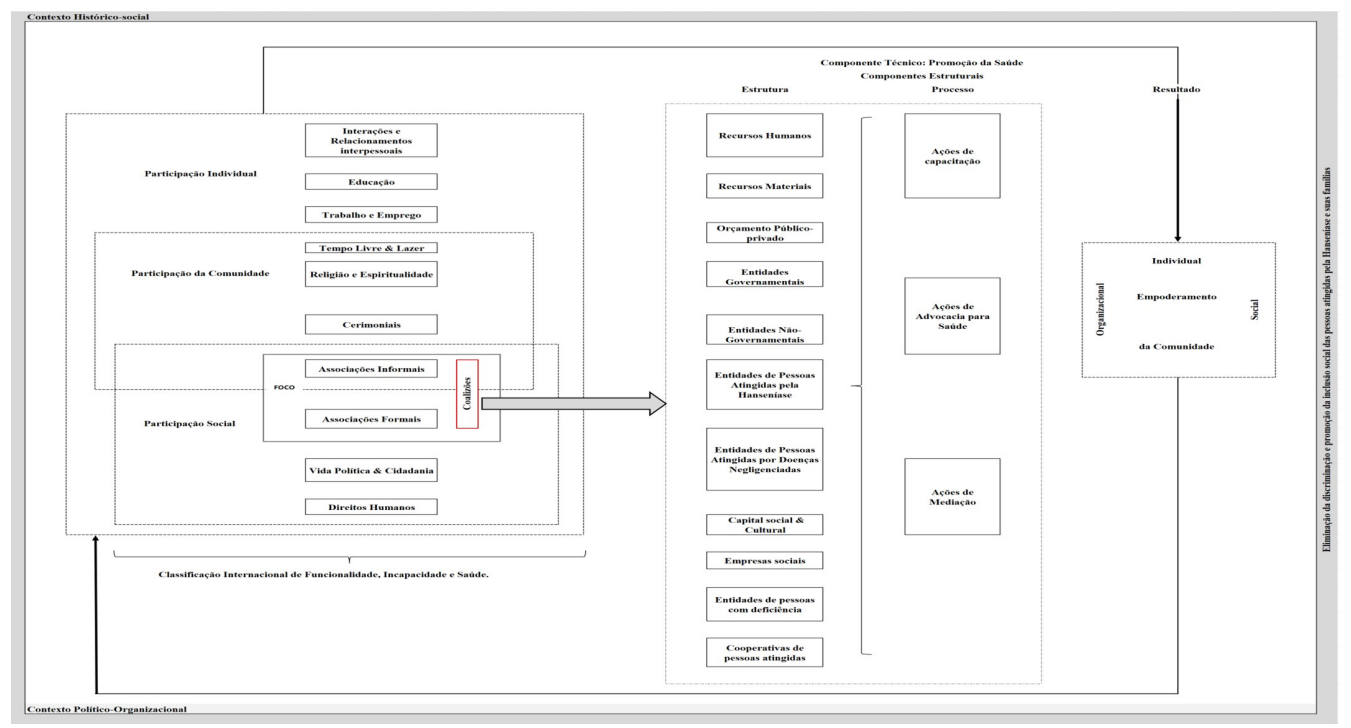

Figura 4. Modelo Lógico Operacional Formação de Coalizões como estratégia de participação para o empoderamento das pessoas atingidas pela Hanseníase. 
Quadro 1. Síntese das críticas, recomendações e questionamentos aos MLO sobre Empoderamento e Participação Social, Coalizões de pessoas atingidas pela Hanseníase.

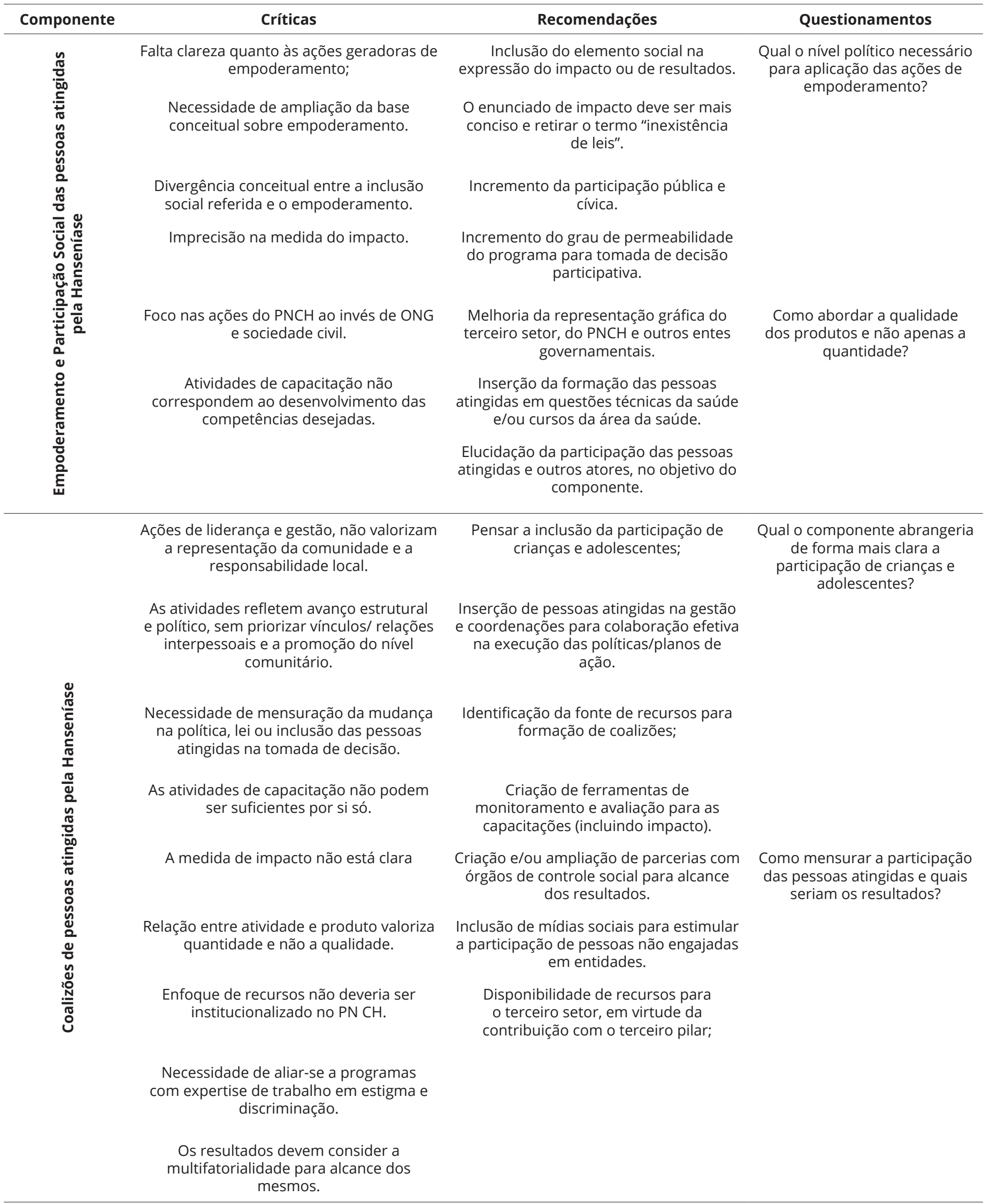


foram adequadas para o empoderamento a nível individual, da comunidade, organizacional e social?", c) "Qual o contexto histórico-social e político-organizacional em que a coalizão foi implementada?", e) "Qual o grau de implementação da coalizão de pessoas atingidas pela Hanseníase no Brasil?" e f) "De que maneira o contexto influenciou na implementação da coalizão de pessoas atingidas pela Hanseníase como estratégia de participação para o empoderamento?".

Os resultados da rodada foram apresentados para os stakeholders na terceira rodada em formato de relatório curto. A análise do papel e interesse dos stakeholders como objetivo não foi alcançada neste momento e foi sugerido que este processo seja realizado na construção do Modelo Teórico da Avaliação. Por este ser um EA utilizando os princípios da avaliação de empoderamento, vale ressaltar que a participação não encerrou com a apresentação dos resultados e críticas ao modelo, a terceira rodada como devolutiva foi um marco operacional para o estudo.

\section{Discussão}

Este Estudo de Avaliabilidade utilizou o método do Painel Delphi Policy para facilitar e oportunizar a participação e envolvimento dos stakeholders. A consulta aos interessados foi pautada nos princípios da inclusão, da participação democrática, do conhecimento da comunidade e da capacitação, propostos por Fetterman (Fetterman, 2015), em prol da elaboração de futura pesquisa avaliativa com abordagem para o empoderamento das pessoas atingidas pela Hanseníase.

No campo da avaliação, a Teoria do Empoderamento integra percepções de controle (dos processos aos resultados), consciência crítica e participação, o que permite aos indivíduos e comunidades desempenhar um papel e agir de modo ativo na transformação (Fetterman, 2017; Fetterman et al., 2014). A autodeterminação é um conceito subjacente a esta teoria e visa imprimir a capacidade de tomada de decisão sobre os processos da vida, o que inclui a necessidade de fazer escolhas racionais dos melhores caminhos e das alternativas possíveis (Fetterman, 2017).

A Avaliação de Empoderamento integra um conjunto de abordagens construtivistas da quarta geração, cujo princípio é a negociação através do diálogo e participação dos atores envolvidos na intervenção (Couto et al., 2019). Neste tipo de avaliação os participantes controlam todas as etapas da avaliação e o avaliador atua como um facilitador do processo avaliativo, na capacitação técnica e crítica dos envolvidos (Fetterman, 2017; Fetterman et al., 2014; Santos et al., 2018).

Os stakeholders foram tomadores de decisão quanto ao objeto (componente que será avaliado) e perguntas da pesquisa avaliativa que será realizada. A valoração dos componentes foi realizada através de técnica simples de ranqueamento. A priorização, sem critérios prédefinidos pelas pesquisadoras, permitiu à captação das disputas de interesse no contexto sócio-político e de financiamento de intervenções e pesquisas relacionadas ao combate a discriminação e promoção da inclusão das pessoas atingidas pela Hanseníase.

A seleção das entidades e representações, tanto nacionais quanto internacionais, foi viabilizada pela inserção de uma das pesquisadoras no contexto de representação das pessoas atingidas pela Hanseníase em ambos os cenários. Moreau (2017) afirma que não há um procedimento conclusivo para a seleção das partes interessadas, no entanto é importante selecionar indivíduos que representam de fato os interesses da comunidade e dos membros do programa. Apesar da articulação e opção por tomadores de decisão, o maior absenteísmo nas respostas se deu por parte de representantes de organismos internacionais.

Estes eventos remetem a necessidade de análise de contexto associada aos estudos de avaliabilidade com abordagem para o empoderamento. Chouinard \& Milley (2016) identificam o contexto como "um fenômeno complexo, multifacetado e interativo que abrange aspectos sociais e históricos, com dimensões políticas, ecológicas e culturais". Para estes autores, compreender o 
espaço do contexto possibilita a interação relacional entre avaliadores e as partes interessadas na avaliação.

O reconhecimento do contexto e das relações de poder envolvidas tornam-se desafios para avaliadores de empoderamento, que buscam trabalhar com programas de saúde pública projetados internacionalmente. Chi et al. (2018) apresentam uma crítica ao modo de planejamento, monitoramento e avaliação deste tipo de intervenção, principalmente, no que tange às questões de financiamento e o modo de avaliá-las pautado em um modelo tecnocrático e biomédico, que não considera a autodeterminação e a apropriação pela comunidade.

Facilitar processos e estimular a participação dos interessados na avaliação não é uma tarefa simples. Fetterman (2019) chama o avaliador de empoderamento de "amigo crítico", visto que, por acreditar no objetivo da intervenção, ele deve ser capaz de fornecer informações baseadas em evidências, de modo crítico-reflexivo e diplomático. O acolhimento dos questionamentos, das críticas e sugestões quanto à formulação dos MLO dos componentes do III pilar da Estratégia Global e a devolução por meio de relatório com a sistematização das informações permitiu aos stakeholders e avaliadores a reflexão sobre as questões de linguagem e competência cultural.

Duas questões se destacam quanto à linguagem. A primeira diz respeito ao uso do inglês como língua universal. Vimos que a tradução dos instrumentos, relatórios e das figuras dos MLO, por si só não foi suficiente para facilitar a compreensão por parte de alguns envolvidos. O uso de linguagem ou jargões técnico-científico, com não especialistas ou que não possuem o inglês como língua mãe, contribuiu para a ausência de respostas em alguns momentos. Moreau (2017) sugere o uso de ferramentas tecnológicas para apoiar uma série de barreiras à avaliação quando os stakeholders não estão geograficamente reunidos, incluindo a acessibilidade a materiais para o treinamento e repasse das informações em linguagem adequada.

A segunda questão diz respeito à competência cultural e expertise sobre determinados temas pelos envolvidos. Na primeira rodada do painel, as respostas ao componente "Empoderamento e Participação Social das pessoas atingidas pela Hanseníase" variaram de acordo com a base teórico-conceitual, interpretação e aplicação dos termos e/ou conceitos de "empoderamento", "participação da comunidade" e "participação social".

Empoderamento e participação são conceitos que foram associados ao campo da Promoção da Saúde. As estratégias prioritárias deste campo (capacitação, advocacy para saúde e mediação) representam um processo social e político abrangente, onde se busca a transformação de uma realidade, a partir do fortalecimento das capacidades e habilidades dos indivíduos e comunidades (Heidmann et al., 2006). Neste contexto, os termos passaram a ser utilizados na elaboração de intervenções e a promoção vista como um componente técnico de vários programas da saúde.

Na segunda rodada, o empoderamento e a participação foram considerados como estruturantes, retroalimentáveis e interdependentes. $O$ alto grau de subjetividade e variações dificulta a proposição de indicadores específicos de resultado a médio e longo prazo. Kuipers (2014), por exemplo, refere que a compreensão, medição e uso do termo empoderamento, para pessoas com deficiência, é diferente entre países em desenvolvimento e desenvolvidos. Enquanto, Baquero (2006) associa para cada nível de empoderamento (individual, organizacional e da comunidade) um determinado foco, onde os indicadores dependem da área de atuação e da intervenção propriamente dita.

Esta percepção foi possível através do exercício da modelização dos componentes e do entendimento da formação de coalizão como estratégia de intervenção para o fortalecimento da participação e do empoderamento. O resultado da primeira rodada demonstrou que o modelo pipeline elaborado, de acordo com os passos de Bezerra et al. (2010), não correspondia à lógica operacional dos componentes eleitos.

Champagne et al. (2011a) referem que a modelização tem por objetivo representar tanto o problema a ser corrigido e suas causas quanto o percurso entre as causas imediatas e distantes. Deste modo, a construção lógica da intervenção junto aos stakeholders é fundamental para 
que o processo avaliativo corresponda ao princípio de melhoria do programa, proposto pela abordagem de empoderamento (Fetterman, 2015).

O MLO da "Formação de coalizões de pessoas atingidas pela Hanseníase" parte da premissa do construtivismo social. Para Conrad \& Barker (2011) indivíduos e grupos contribuem para a produção do conhecimento e de uma realidade social percebida. Do mesmo modo, determina a participação das pessoas atingidas pela hanseníase para produção do empoderamento individual, da comunidade, organizacional e social, através da formação de coalizões.

Anderson et al. (2015) definem coalizão em saúde como uma estratégia que visa a redução das disparidades na saúde, através da mobilização de representantes (de populações-alvo, cidadãos e profissionais de diferentes áreas, organizações públicas e privadas de múltiplos setores da sociedade), que atuam em caráter de voluntariado, em diferentes níveis (do local ao global), no planejamento de intervenções (como programas e/ou políticas), a partir da identificação de problemas comuns e tomada de decisão sobre questões complexas em saúde.

Pensar a formação de coalizões no âmbito do componente técnico Promoção da Saúde, em Programas de Controle da Hanseníase, foi uma estratégia para dar visibilidade a esta intervenção, visto que elas podem ser completamente independentes desta situação políticoorganizacional. Outrossim, o EA mostrou a necessidade de compreender o papel das coalizões, as atividades desenvolvidas por elas no campo da promoção da saúde, as relações entre os atores e os resultados deste processo.

Entre os limites do uso do Painel Delphi Político para este tipo de EA também se destacaram: o tamanho e formato de apresentação do painel; o quantitativo de informações a serem analisadas tornando exaustivo e proporcionando o desinteresse de alguns stakeholders ao longo de sua execução; a análise por componente técnico para chegar ao uso de dois elementos e o estímulo a participação dos atores durante todo o estudo devido ao cronograma de ações. No entanto, a apropriação pelos stakeholders do processo avaliativo desde sua concepção e a reflexão sobre cada um dos componentes foi um dos ganhos deste estudo.

\section{Conclusão}

A realização deste EA permitiu a elaboração de um Modelo Causal e Modelo Lógico Teórico do terceiro pilar da "Estratégia Global 2016-2020: por um mundo livre de hanseníase", a decisão por avaliar a formação de coalizões de pessoas atingidas pela hanseníase como estratégia de participação para o empoderamento, a escolha das perguntas avaliativas sobre a implementação de coalizão e a elaboração do MLO orientador do processo avaliativo, considerando a abordagem da avaliação de empoderamento.

Vale destacar a ocorrência do mesmo quantitativo de respostas por organizações nacionais e internacionais, ou seja, três de cada. No entanto, houve maior representatividade das respostas a nível nacional com cinco participantes nacionais e quatro internacionais, para a segunda rodada. Através do uso da abordagem de empoderamento no estudo de avaliabilidade apreende-se que os avaliadores precisam estar atentos a seleção dos melhores representantes das partes interessadas, ao uso adequado de ferramentas de comunicação disponíveis para apoiar os processos e a tomada de decisão coletiva, ao estímulo cotidiano à participação, a necessidade de análise de contexto e das relações de poder que permeiam as intervenções e as partes interessadas, a necessidade da diplomacia e autoconhecimento quando se integra o constructo da intervenção em ambos os papéis (avaliador e stakeholder).

Acredita-se assim que para os especialistas representantes das organizações governamentais e não-governamentais, em especial das entidades de pessoas atingidas, este estudo permitiu a visualização de processos através da estruturação lógica do terceiro pilar. Além de apoiar o planejamento para futuras intervenções na área do combate a discriminação e promoção da inclusão por parte de governos e a reinvindicação de direitos através da participação social organizada em coalizões de pessoas atingidas pela hanseníase.

Ademais, a necessidade de reflexão sobre sucessos e fracassos nos caminhos adotados pelos Programas de Controle da Hanseníase, incluindo a participação efetiva das pessoas 
atingidas na formulação das intervenções do nível global ao local. E por último, a compreensão do processo de formação, implementação e papel destas coalizões, como estratégia de participação para o empoderamento a nível individual, comunitário, organizacional e social.

\section{Fonte de financiamento}

Não há.

\section{Conflito de interesse}

Não há.

\section{Referências}

Anderson, Laurie, Adeney, Kathryn, Shinn, Carolynne, Safranek, Sarah, Buckner-Brown, Joyce, \& Krause, Kendall. (2015). Community coalition-driven interventions to reduce health disparities among racial and ethnic minority populations. Cochrane Database of Systematic Reviews, (6), CD009905. http://dx.doi. org/10.1002/14651858.CD009905.pub2

Araujo, Inesita, Moreira, Adriano, \& Aguiar, Raquel. (2012). Doenças negligenciadas, comunicação negligenciada: Apontamentos para uma pauta política e de pesquisa. Revista Eletrônica de Comunicação, Informação e Inovação em Saúde, 6(4).

Baquero, Rute Vivian Angelo. (2006). Empoderamento: Questões conceituais e metodológicas. Redes, 11(2), 77-93.

Baratieri, Tatiane, Nicolotti, Célia Adriana, Natal, Sonia, \& Lacerda, Josimari Telino de. (2019). Aplicação do estudo de avaliabilidade na área da saúde: Uma revisão integrativa. Saúde em Debate, 43(120), 240-255 http://dx.doi.org/10.1590/0103-1104201912018

Bezerra, Luciana, Cazarin, Gisele, \& Alves, Cinthia. (2010). Modelagem de programa: Da teoria à operacionalização. In Isabella Samico, Eronildo Felisberto, Ana C. Figueiró \& P. Frias (Eds.), Avaliação em saúde: Bases conceituais e operacionais (pp. 65-78). Rio de Janeiro: Medbook.

Brasil. Ministério da Saúde. (2020). Boletim Epidemiológico Hanseníase 2020. Brasília: MS/CGDI. Recuperado em 27 de julho de 2021, de http://www.aids.gov.br/pt-br/pub/2020/boletim-epidemiologico-dehanseniase- 2020

Brousselle, Astrid, Champagne, François, Contandriopoulos, André-Pierre, \& Hartz, Zulmira. (2011). A análise de implantação. In Astrid Brousselle, François Champagne, André-Pierre Contandriopoulos \& Zulmira Hartz (Eds.), Avaliação: Conceitos e métodos (22. ed., pp. 217-238). Rio de Janeiro: Fiocruz.

Brunner, Richard, Craig, Peter, \& Watson, Nick. (2019). Evaluability assessment: An application in a complex community improvement setting. Evaluation, 25(3), 349-365. PMid:31379464. http://dx.doi. org/10.1177/1356389019852126

Champagne, François, Brousselle, Astrid, Hartz, Zulmira, \& Contandriopoulos, André-Pierre. (2011a). Avaliação: Conceitos e métodos: Modelizar as intervenções (pp. 61-74). Rio de Janeiro: Editora Fiocruz.

Chi, Chunhuei, Tuepker, Anaïs, Schoon, Rebecca, \& Núñez Mondaca, Alicia. (2018). Critical evaluation of international health programs: Reframing global health and evaluation. The International Journal of Health Planning and Management, 33(2), 511-523. PMid:29314258. http://dx.doi.org/10.1002/hpm.2483

Chouinard, Jill, \& Milley, Peter. (2016). Mapping the spatial dimensions of participatory practice: A discussion of context in evaluation. Evaluation and Program Planning, 54, 1-10. PMid:26476858. http:// dx.doi.org/10.1016/j.evalprogplan.2015.09.003

Conrad, Peter, \& Barker, Kristin. (2011). A construção social da doença: Insights-chave e implicações para políticas de saúde. Idéias, 2(2), 183-219.

Couto, Felipe, Carrieri, Alexandre, \& Ckagnazaroff, Ivan. (2019). Participação na avaliação de políticas públicas: A pesquisa construtivista e a quarta geração de avaliação. Gestão \& Planejamento., 20(1), 36-55. http://dx.doi.org/10.21714/2178-8030gep.v.20.5141

Dean, Laura, Tolhurst, Rachel, Nallo, Gartee, Kollie, Karsor, Bettee, Anthony, \& Theobald, Sally. (2019). Neglected tropical disease as a 'biographical disruption': Listening to the narratives of affected persons to develop integrated people centred care in Liberia. PLoS Neglected Tropical Diseases, 13(9), e0007710. PMid:31490931. http://dx.doi.org/10.1371/journal.pntd.0007710

FETTERMAN, D. et al. Collaborative, Participatory, and Empowerment Evaluation: Building a Strong Conceptual Foundation for Stakeholder Involvement Approaches to Evaluation (A Response to Cousins, Whitmore, and Shulha, 2013). American Journal of Evaluation, v. 35, n. 1, p. 144-148, mar. 2014.

Fetterman, David, \& Wandersman, Abraham. (2017). Celebratory reflections, appreciations, clarifications, and comments. Evaluation and Program Planning, 63, 143-146. PMid:27993390. http://dx.doi.org/10.1016/j. evalprogplan.2016.12.006 
Fetterman, David. (2015). Empowerment evaluation. In James D. Wright (Ed.), International Encyclopedia of the Social \& Behavioral Sciences (pp. 577-583). Amsterdam: Elsevier. http://dx.doi.org/10.1016/B978-008-097086-8.10572-0

Fetterman, David. (2017). Transformative empowerment evaluation and freirean pedagogy: Alignment with an emancipatory tradition: Transformative empowerment evaluation and freirean pedagogy. New Directions for Evaluation, 2017(155), 111-126. http://dx.doi.org/10.1002/ev.20257

Fetterman, David. (2019). Empowerment evaluation: A stakeholder involvement approach. Health Promotion Journal of Australia, 30(2), 137-142. PMid:30972895. http://dx.doi.org/10.1002/hpja.243

Heidmann, Ivonete, Almeida, Maria Cecília Puntel de, Boehs, Astrid Eggert, Wosny, Antonio de Miranda, \& Monticelli, Marisa. (2006). Promoção à saúde: Trajetória histórica de suas concepções. Texto \& Contexto Enfermagem, 15(2), 352-358. http://dx.doi.org/10.1590/S0104-07072006000200021

Kuipers, Pim. (2014). Empowerment in community-based rehabilitation and disability-inclusive development. Disability, CBR and Inclusive Development, 24(4), 24-42. http://dx.doi.org/10.5463/dcid. v24i4.274

Lastória, Joel Carlos, \& Abreu, Marilda Aparecida Milanez Morgado de. (2014). Leprosy: Review of the epidemiological, clinical, and etiopathogenic aspects: Part 1. Anais Brasileiros de Dermatologia, 89(2), 205-218. PMid:24770495. http://dx.doi.org/10.1590/abd1806-4841.20142450

Marques, Joana Brás Varanda, \& Freitas, Denise de. (2018). Método DELPHI: Caracterização e potencialidades na pesquisa em educação. Pro-Posições, 29(2), 389-415.

Moreau, Katherine A. (2017). Twelve tips for planning and conducting a participatory evaluation. Medical Teacher, 39(4), 334-340. PMid:28379083. http://dx.doi.org/10.1080/0142159X.2017.1286310

Nery, Joilda, Ramond, Anna, Pescarini, Julia, Alves, André, Strina, Agostino, Ichihara, Maria, Fernandes Penna, Maria, Smeeth, Liam, Rodrigues, Laura, Barreto, Mauricio, Brickley, Elizabeth, \& Penna, Gerson. (2019). Socioeconomic determinants of leprosy new case detection in the 100 Million Brazilian Cohort: A population-based linkage study. The Lancet. Global Health, 7(9), e1226-e1236. PMid:31331811. http:// dx.doi.org/10.1016/S2214-109X(19)30260-8

Oliveira, Verônica Macário de, Correias, Suzanne Erica Nóbrega, \& Gomezs, Carla Regina Pasa. (2018). Policy Delphi as a research method for framework development: The case of the roles of promoting sustainable consumption in the brazilian context. Revista Capital Científico Eletrônica., 16(2), http://dx.doi. org/10.5935/2177-4153.20180011

Organização Mundial de Saúde - OMS. (1986). Carta de Ottawa. Genebra: OMS. Recuperado em 27 de julho de 2021, de https://bvsms.saude.gov.br/bvs/publicacoes/carta_ottawa.pdf

Organização Mundial de Saúde - OMS. (2013). Manual prático da CIF. Genebra: OMS. Recuperado em 27 de julho de 2021, de http://www.fsp.usp.br/cbcd/wp-content/uploads/2015/11/Manual-Pra\%CC\%81 ticoda-CIF.pdf

Pescarini, Julia Moreira, Strina, Agostino, Nery, Joilda Silva, Skalinski, Lacita Menezes, Andrade, Kaio Vinicius Freitas de, Penna, Maria Lucia F, Brickley, Elizabeth B, Rodrigues, Laura C, Barreto, Mauricio Lima, \& Penna, Gerson Oliveira. (2018). Socioeconomic risk markers of leprosy in high-burden countries: A systematic review and meta-analysis. PLoS Neglected Tropical Diseases, 12(7), e0006622. PMid:29985930. http:// dx.doi.org/10.1371/journal.pntd.0006622

Ribeiro, Mara Dayanne Alves, Silva, Jefferson Carlos Araujo, \& Oliveira, Sabrynna Brito. (2018). Estudo epidemiológico da hanseníase no Brasil: Reflexão sobre as metas de eliminação. Revista Panamericana de Salud Pública, 1-7. http://dx.doi.org/10.26633/RPSP.2018.42

Santos, Elizabeth Moreira dos, Oliveira, Egleubia Andrade de, Cardoso, Gisela Cordeiro Pereira, Cavalcanti, Maria de Lourdes Tavares, Costa, Antônio José Leal, \& Câmara, Volney. (2018). Evaluaciones inclusivas: Una reflexión sobre las posibilidades e implicaciones de enfoques participativos para la práctica evaluativa. Revista de Salud Ambiental, 18(2), 172-181.

World Health Organization - WHO. (2011). Guidelines for strengthening participation of persons affected by leprosy in leprosy services. Genebra.

World Health Organization - WHO. (2016). Estratégia global para hanseníase 2016-2020: Aceleração rumo a um mundo sem hanseníase. Genebra: World Bank Publications.

World Health Organization - WHO. (2017a). Estratégia global para hanseníase 2016-2020: Aceleração rumo a um mundo sem hanseníase. Guia de monitoramento e avaliação. Nova Deli: Organização Mundial da Saúde, Escritório Regional para o Sudeste Asiático.

World Health Organization - WHO. (2017b). Estratégia mundial para Hanseníase 2016-2020: Aceleração rumo a um mundo sem hanseníase. Manual operacional. Genebra.

World Health Organization - WHO. (2020). Global leprosy update, 2018: Moving towards a leprosyfree world. Genebra: WHO Leprosy. Recuperado em 27 de julho de 2021, de https://apps.who.int/iris/bitstream/ handle/10665/326775/WER9435-36-en-fr.pdf?ua=1

World Health Organization - WHO. (2021). Global health observatory data repository. Genebra: WHO Leprosy. Recuperado em 27 de julho de 2021, de https://apps.who.int/gho/data/node.main.A1638?lang=en 